\title{
Norma negoziale e paradigma normativo *
}

\author{
Luigi Bagolini \\ Catedrático de F'ilosofia do Di- \\ reito na Faculdade de Direito da \\ Universidade de Gênova
}

\begin{abstract}
Sommario: 1. Problema: come debba essere pensata la norma giuridica perchè si possa coerentemente pensare alla essenza normativa del negozio. 2. Negozio e promessa. L'assurdo inerente al concetto di autolimitazione della volontà: impossibilità di uscime attraverso la nozione di affidamento. 3. Autolimitazione della volontà ed autocomando. La critica del'imperatività della norma giuridica in quanto necessaria alla dottrina normativa del negozio. “Inversione' operata da Kelsen nella rappresentazione tradizionale del paradigma normativo. 4. Inaccettabilità della "inversione" kelseniana. 5. Determinazione di un paradigma della normatività rispetto al quale sia coerente affermare l'essenza normativa dei negozi. 6. Norma e sanzione. Vantaggi offerti dall'ipotesi qui prospettata in confronto con $\mathrm{i}$ risultati delle dottrine criticate.
\end{abstract}

1. Come deve essere pensata in generale la norma giuridica perchè si possa coerentemente pensare alla essenza normativa e quindi al contenuto precettivo del negozio? Il problema espresso da questa domanda non è di certo soltanto apparente. Infatti a seconda dei caratteri che si attribuiscano in generale alla norma giuridica, sarà o non sarà possibile parlare coerentemente di una norma giuridica negoziale. C'è qui una questione di coerenza del lin-

* Relazione presentata al IV congresso nazionale italiano di filosofia del diritto (Pavia, ottobre 1959). 
guaggio giuridico in rapporto al paradigma della normatività più o meno esplicitamente presente nel discorso di chi sostenga l'essenza normativa del negozio ${ }^{1}$. Il che naturalmente non significa che una concezione normativa del negozio sia utilmente accettabile per il solo fatto della sua coerenza rispetto al paradigma della normatività in essa implicato.

Indubbiamente nel pensiero dei più coerenti e consapevoli assertori della concezione normativa del negozio deve essere presente un modo diverso di concepire -- e direi quasi anche di sentire - il paradigma della normatività rispetto ai sostenitori delle concezioni cui essi si oppongono. In definitiva la critica della concezione consensualistica del contratto, la critica della concezione volontaristica del negozio, la critica della volontà come fondamento della obblígatorietà delle promesse, tutte queste critiche ${ }^{2}$ sembrano mosse, rispetto alle concezioni criticate, da tutta una diversa consapevolezza della realtà giuridica nelle sue concrete ed attuali determinazioni, e quindi di conseguenza anche da una diversa rappresentazione del paradigma normativo, la quale si manifesta in varie maniere più o meno evidenti e più o meno chiare a seconda dei diversi autori. D'altra parte - giova appena notare - le variazioni nel modo di concepire il paradigma della normatività sono senza dubbio influenzate dal movimento, dalla trasformazione e dalla insorgenza storica di valori, finalità, interessi,

1. Vedi anche per i riferimenti alla vasta letteratura E. BETTI, Teoria generale del negozio giuridico ${ }^{2}$, Torino, 1955; SAlvatore Romano, Autonomia privata, Milano, 1957; L. FERRI, L'autonomia privata, Mila no, 1959.

2. Vedi ad esempio in una vasta letteratura G. Asturr. I principi fondamentali dei contratti nella storia del diritto italiano in Annali di storia del diritto, I, 1957, pp. 13-42. e, fra le più varie prospettive, G. MoRIN, La désagrégation de la théorie contractuelle du code; $\mathbf{M}$. DJUVARA, L'idée de convention et ses manifestations juridiques, F. BATTAGLIA, Le problème du contrat in Archives de philosophie du droit et de sociologie juridique, 1940 , rispettivamente alle pp. 7-32; 110-158; 174-185. Vedi pure M. VILLEY, Essor et décadence du volontarisme juridique in Archives de philosophie du droit, 1957, pp. 87-98. 
ideologie, strutture ambientali sociali ed economiche cui si riconnettono trasformazioni della cosidetta coscienza giuridica ${ }^{3}$ sia pure semplicemente intesa come modo di sentire, di vivere e di concepire di volta in volta le funzioni e quindi anche le strutture dei vari ordinamenti giuridici.

2. Per tentare di rispondere alla proposta domanda: come debba essere pensata la norma giuridica perchè si possa coerentemente pensare alla essenza normativa del negozio, bisogna tenere anzitutto presente qualcuna delle ragioni più rilevanti per cui anche agli occhi dei sostenitori più coerenti della concezione normativa, non sembra accettabile una concezione volontaristica del negozio. Ciò proprio per poi determinare come il paradigma normativo debba essere pensato affinché nella concezione normativa del negozio non si ripresentino surrettiziamente quelle medesime difficoltà che siano rilevabili nella concezione volontaristica.

Dai sostenitori della dottrina volontaristica il negozio è concepito come manifestazione od anche come dichiarazione di volontà che mira a produrre effetti giuridici (e ciò nonostante l'interno dissidio fra i sostenitori del così detto dogma della volontà ed i sostenitori del così detto dogma della dichiarazione ${ }^{4}$ ). L'essenza del negozio é vista

3. Cfr. B. Paradisi, Le dogme de l'histoire vis-à-vis de l'historiographie juridique, in Archives de philosophie du droit, 1959, p. 25 e ss.

4. In sostanza, nonostante le discussioni fra i così detti sostenitori del dogma della volontà ed i sostenitori del dogma della dichiarazione, anche i sostenitori del dogma della dichiarazione possono essere considerati nell'ambito di una concezione volontaristica del negozio intesa in senso lato. Como dice P. ROUBIER, (Essai sur la responsabilité précontractuelle Lione, 1911, p. 269 e ss.; Le rôle de la volonté dans la création des droits et des devoirs in Archives de philosophie du droit, 1957 , p. 16), non è necessario negare alla volontà la sua funzione creatrice negli atti giuridici per soddisfare quegli interessi delle relazioni giuridiche $\mathbf{i}$ quali esigono che $\mathrm{i}$ contraenti possano contare ciascuno rispettivamente sulla volontà dichiarata dall'altro. In effetti è sufficiente dire che la dichiarazione costituisce il modo di espressione della volontà e che non è necessario obbligare ciascuna delle parti a provare la 
nella volontà. Alla volontà è attribuito il potere di creare effetti giuridici e quindi anche di far nascere, modificare od estinguere rapporti o situazioni preliminari a rapporti. ${ }^{5}$. Ma come si spiega che alla volontà di obbligarsi giuridicamente la legge positiva faccia seguire un vincolo giuridico?

Riferiamoci agli atti patrimoniali fra vivi, e specificamente agli atti di obbligazione distinti dagli atti di disposizione ${ }^{6}$, e quindi alle promesse. Tutte le volte che in un atto è implicato, in una maniera o nell'altra, il fenomeno

corrispondenza della sua dichiarazione con la sua volontà. Secondo Roubier, questo è del resto il punto di vista del diritto positivo francese il quale non ammette che una nullità relativa in materia di errore, e soltanto, d'altra parte, l'ammette in certi casi di errore.

Secondo E. BETTI (op. cit., pp. 56-57) quando per "volontà" dichiarata non si intenda "statuizione o precetto dell'autonomia privata", si finisce in un equivoco. "O si cade nell'errata opinione che la dichiarazione, anziché servire a costituire e porre in essere un precetto dell'autonomia privata, serva unicamente a rilevare uno stato d'animo, a discoprire un fatto psichico interno - il fatto del volere - e che in questo fatto psichico, per sé incontrollabile, consista e si esaurisca l'essenza del negozio. $\mathrm{O}$ comunque si configura la volontà come un'entità a se stante che si contrappone e si abbina alla dichiarazione conservandosi ad essa superiore come l'anima al corpo e, senza perdere la propria netta indipendenza da essa, trova in essa un semplice complemento e un mezzo di rivelazione"

Non essendo qui possibile fare riferimento a tutta la più importante e ben vasta letteratura critica sulla concezione volontaristica, rimando anche per i riferimenti bibliografici, dal punto di vista della concezione precettistica, alla citata opera fondamentale di Betti.Vedi pure il citato libro di L. FgRRI su L'autonomia privata, in cui la tesi precettistica è sostenuta e dimostrata nelle sue più rilevanti implicazioni.

5. Vedi anche per i riferimenti alla letteratura A. LEVI, Teoria generale del diritto, Padova, 1950, p. 303 e ss.

6. "Gli atti patrimoniali fra vivi" "si distinguono in atti d'obbligazione (promesse) e atti di disposizione". "Gli atti di obbligazione sono quelli che hanno lo scopo o funzione di dar luogo ad un'obbligazione: in particolare, i contratti. Gli atti di disposizione sono quelli che hanno lo scopo o funzione di disporre di un diritto patrimoniale". Così G. GORLA, Il potere della volontà nella promessa come negozio giuridico in Riv. dir. comm., 1956, p. 21. 
della promessa ${ }^{7}$, che cos'è che la legge tutela: la volontà del promittente di autoobbligarsi o l'affidamento del promissario? Questi interrogativi mettono la dottrina volontaristica di fronte a difficoltà che sembrano veramente insuperabili. La volontà del promittente di autoobhligarsi rimanda al concetto di autolimitazione della volontà.

Tale concetto appare da vari punti di vista assurdo. Infatti la volontà presente che si autolimita è una volontà presente che ha come oggetto una volontà futura, è un volere-volere. Ma il volere-volere non è volere concreto e reale, è volontà irreale. La volontà del promittente di autoobbligarsi è quindi un presupposto irreale. Vale qui ciò che il Croce ha dimostrato: che "si vuole soltanto in concreto, ossia in una situazione determinata" ed in modo tale

7. A. ReINACH: (Zur Phänomenologie des Rechts. Die apriorischen grundlagen des bürgelichen Rechts, Monaco, 1953 - già apparso nel 1913 in Jahrbuch für Philosophie und phänomenologische Forschung col titolo: Die apriorischen Grundlagen des bürgerlichen Rechts - p. 84) non vuole dare una vera e propria teoria della promessa. La promessa implica di per se stessa un obbligo e una pretesa. Volere spiegare come e perché una promessa sia obbligatoria sarebbe per Reinach come volere spiegare la ragione per cui $1 \times 1=1$.

Sotto questo aspetto la posizione di Reinach è assiomaticistica. L'obbligatorietà della promessa è un assioma suscettibile soltanto di essere intuito e che quindi è inutile cercare di spiegare. É escluso il problema concernente il perché e le condizioni di possibilità del costituirsi di un obbligo nei confronti del promittente. Esclusa ogni spiegazione come mediazione in quanto che l'obbligatorietà della promessa resta una verità immediata.

Non ci si può riferire alla particolarità della posizione di Reinach il quale ha voluto utilizzare nel suo studio il metodo fenomenologico (così come si rivela delineato fra l'altro nel suo scritto: "Was ist Phänomenologie?", ristampa, Monaco, 1951) per arrivare a determinare gli elementi fondamentali di una dottrina aprioristica del diritto - in cui si tratta poi di vedere in che cosa chiaramente consista l'apriorità.

La concezione della obbligatorietà della promessa come verità assiomatica si trova ribadita in altri autori contemporanei di formazione diversa rispetto al Reinach come, ad esempio da D. Ross in Foundations od Ethics (Oxford, 1939, p. 77), secondo cui l'obbligatorietà della promessa è un assioma senza del quale nesun ordine morale sarebbe concepibile. 
.che il volere "si traduca immediatamente in azione, cioè che sia insieme azione effettiva" 8 .

"L'anno scorso ho promesso e stipulato perchè volevo. Ora non voglio più". "Perchè" "dev'essere la volontà passata, morta e non più mia" che dispone della mia volontà presente ${ }^{9}$ ?

Già Hume contro la concezione giusnaturalistica della volontà assunta come fondamento della obbligatorietà delle promesse aveva spiegato molto chiaramente la ragione per cui, secondo il suo modo di esprimersi, "l'atto" "che entra in una promessa e produce la sua obbligatorietà" non può essere né "il desiderio né la volontà" di compiere una particolare azione. Mentre "una promessa si riferisce sempre al futuro", "la volontà ha soltanto una influenza sulle azioni presènti" 10 .

8. B. CROCE, Filosofia della pratica ${ }^{6}$ Bari, 1950 , p. 33 e ss., p. 328 .

9. Cfr. G. ReNSI, Lineamenti di filosofia scettica. ${ }^{2}$ Bologna, 1921, p. 165 e ss.

10. Vedi D. Hume, A Treatise of Human Nature, III, 2, 5, ediz. a cura di L. A. Selby-BigGe (Oxford, 1946), p. 516 e in proposito il mio saggio: Esperienza giuridica e politica nel pensiero di D. Hume, estr. dagli Studi senesi, 1947-1948 - vol. LX-LXI (vol. XXXV-XXXVI della II serie) -, p. 54 e ss.

Le argomentazioni di Hume sono in parte riprese da W. SCHUPPE (Grundzüge der Ethik und Rechtsphilosophie, Breslau 1881, p. 304 e ss.) Anche secondo Schuppe l'obbligatorietà della promessa non può essere fondata sulla volontà poichè, come egli dice, il concetto di volontà - e di dichiarazione del volere - non può comprendere il carattere dell'immutabilità. La parola volontà indica il "movimento" nel "contenuto della coscienza" umana; "nessuno può sapere ciò che vorrà, ma soltanto può sapere ciò che ha voluto e che presentemente vuole". "La volontà segue evidentemente le opinioni e - i sentimenti; e quanto poco questi ultimi sono immutabili, tanto poco può esserlo la volontà". Ai sentimenti ed alla volontà non si applica in alcun modo il concetto di obbligo (ib., p. 304).

"La irrevocabilità di una volontà manifestata pù̀ soltanto essere richiesta a favore della sicurezza della proprietà. La obbligatorietà dei contratti - e delle promesse - non consiste in altro che nel significato della volontà obbiettiva del diritto - positivo - la quale, per T'appunto, vuole la irrevocabilità" (ib., p. 305), Con questa sua soIuzione del problema (per la quale si distacca da Hume a cui inizial- 
Il concetto di affidamento del promissario alla volontà del promittente non basta, come sembrano credere alcuni autori, a spiegare il fondamento della obbligatorietà di una promessa e la ragione della sua tutela giuridica evitando le difficoltà, ora accennate, inerenti al concetto di autolimitazione. Nel discorso di chi pur sempre presupponga che oggetto dell'affidamento sia la volontà del promittente, si ripresenta necessariamente l'assurdo dell'autolimitazione della volontà. Il promissario cioè può affidarsi - e calcolare sulla - volontà del promittente soltanto in quanto il promittente abbia autolimitato la sua futura possibilità di volere col mettere, per così dire, la sua volontà a disposizione del promissario. La possibilità dell"affidamento

mente si era avvicinato nella parte critica) Schuppe può essere preso come esempio significativo delle conseguenze a cui può portare logicamente l'impostazione volontaristica e cioè l'assunzione iniziale della promessa come essenzialmente riducibile a manifestazione di volontà.

Se nella promessa c'è solo la volontà del promittente, se nella promessa non c'è qualcosa, che, pur essendo posto in essere dalla volontà del promittente, sia inesprimibile attraverso la nozione di volontà del promittente, è logico, come osserva del resto anche ReINACH (Die apriorischen Grundlagen, cit., pp. 78-79), che la giustificazione dell'obbligatorietà della promessa sia cercata al di fuori della promessa. Inoltre, secondo Reinach (ibidem), Schuppe confonde i due concetti di obbligatorietà e di irrevocabilità ed il suo punto di vista, riferendosi soltanto ai rapporti di proprietà, viene ad essere arbitrariamente limitato.

Nel libro di Fr. BASSEnge: Das Versprechen. Ein Beitrag zur Philosophie der Sittlichkeit (Berlino 1930), si trovano convincenti argomentazioni (p. 27 e ss) contro le varie dottrine per le quali rispettivamente il fondamento dell'obbligatorietà delle promesse è visto nella volontà del promittente, è ricondotto al dovere di dire la verità, è considerato come riducibile al valore di un determinato comportamento indipendentemente dalla considerazione del fatto che tale comportamento sia oggetto di una promessa, è determinato dalla utilità dell'adempimento.

Bassenge accetta in parte l'analisi della promessa fatta da Reinach ma vuole integrarla attraverso la ricerca di un principio metafisico (pp. 7-8). Ebbene il principio metafisico assoluto, ed in un eerto senso di per sé evidente, l'interesse sociale e cosmico, "ein kosmisches Interesse", su cui si fonda l'obbligatorietà della promessa, è, secondo l'autore "la libertà dall'illusione", la quale non è a sua volta semplicemente uno dei momenti della libertà morale, ma ne è piuttosto "il momento 
del promissario dipende ancora dal fatto che la volontà attuale del promittente si trovi limitata dalla sua volontà passata $^{11}$. Che poi oggi l'interesse sociale perseguito dal nostro ordinamento giuridico positivo tenda a identificarsi più con la tutela dell'interesse del promissario che con la tutela della volontà del promittente, potrà essere anche una constatazione vera; la quale però servirà a spiegarci, se mai, la ragione politica della tutela e non il suo presupposto e la sua condizione specificamente giuridica.

essenziale" (p. 33). Precisamente nella promessa l'autore vede un elemento essenziale che si trova in tutte le azioni consapevoli e cioè un impegno che l'agente implicitamente assume di fronte agli atri e di fronte a se stesso. Ogni azione implica un impegno di fronte agli altri che bisogna mantenere nel corso stesso dell'azione affinchè non siano tradite le altrui aspettative. In questo senso per l'appunto una promessa che non sia mantenuta, equivale alla creazione di una illusione, così come equivale alla creazione di una illusione un comportamento che non sia coerente e fedele rispetto al suo implicito significato. (ibidem)

Secondo me ciò che qui dice quest'ultimo autore potrà essere in sé e persé anche accettabile, ma non ci offre una prospettiva propriamente utile a rischiarare il campo delle concrete possibili relazioni, antinomie ed alternative che si riconnettono alla promessa. Cercando di ricondurre - come hanno fatto vari altri autori in un modo o nell'altro - l'essenza della promessa ad un criterio universale ed assoluto di valutazione dei comportamenti umani, Bassenge finisce col perdere il significato specifico della promessa. Poiché se è vero che ci può essere un "interesse cosmico" ed universale all'adempimento delle promesse, è anche vero che in certi casi all'obbligo di stare ai patti possono contrapporsi interessi altrettanto fondamentali ed irriducibili. La considerazione dell'obbligo del promittente come fondato su di una norma la quale sia in relazione di correlazione e di subordinazione rispetto all'ordinamento sociale, morale o giuridico di cui fa parte, appare più utile ad una comprensione delle situazioni concrete: più utile di una riduzione del fondamento dell'obbligatorietà della promessa ad un principio assoluto di incondizionata validità.

11. Il punto di vista secondo il quale la obbligatorietà della promessa è vista in funzione di una specie di acquisto della volontà del promittente da parte del promissario è assai diffuso e si trova presente, fra gli altri, in maniera diversa in KANT (ai paragrafi 18-19 dei Metaphisische Anfrangsgründe der Rechtslehre) e in RosminI (ai paragrafi 368, 1090 e 1094 della Filosofia del diritto). 
3. L'assurdo della volontà che vuole se stessa, del voler-volere, della volontà che si autolimita, è l'assurdo stesso di un comando che chi comanda rivolga a se medesimo. Se l'essenza obbligatoria di una promessa fosse costituita dalla volontà del promittente, la promessa implicherebbe in se stessa questo assurdo dell'autocomando. Pertanto, affinché una concezione normativa, e non volontaristica. del negozio non abbia a riprodurre in sé questo assurdo, bisogna che il precetto negoziale possa essere concepito come strutturalmente diverso ed irriducibile rispetto alla volontà di chi lo crea. Bisogna quindi che la norma negoziale possa non essere pensata come imperativo.

- Per la discussione della posizione di questi due autori in un chiaro inquadramento del problema che qui ci interessa, rimando al penetrante saggio di W. CESARINI SForza: Promessa e giuramento in Scritti giuridici in onore di F. Carnelutti, Padova, 1950, v. I, pp. 225227.

Il medesimo punto di vista si trova in Grozio e nei giusnaturalisti. Per la documentazione relativa vedi il fondamentale studio di GoRLA: Il potere dellos volontà nella promessa, cit., pp. 24-27, da considerare in relazione all'opera dell'autore: Il contratto. Problemi fondamentali trattati con il metodo comparativo e casistico (in 2 vol. Milano, 1955). Il Gorla critica con argomenti che mi sembrano molto validi sia il concetto dell'autolimitazione della volontà sia la ridetta tendenza a "parificare" atto di alienazione e promessa (Il potere della volontà nella promessa, cit., p. 25 e ss); ma non mi sembra che il concetto di affidamento proposto dal Gorla (ib., p. 45) possa sottrarsi alle difficoltà inerenti alle dottrine criticate se l'affidamento sia pur sempre semplicemente inteso come affidamento del promissario nei confronti della "volontà" del promittente.

Secondo CeSARINi SFORZA (op. cit.. p. 224) "promettere significa affidare un proprio comportamento possibile (e quindi futuro) alla volontà altrui, ed è la volontà altrui che crea il vincolo obbligatorio, ossia che pone il comportamento possibile in uno stato d'obbligo. Chi accetta la promessa capta, per così dire, l'azione proiettata dal promittente fuori dalla sua sfera volitiva, l'azione che quindi è obiettivata, divenuta un "oggetto" del mondo pratico; e la promessa continua ad obbligare fino a tanto che il destinatario di essa "tiene" nella propria sfera volitiva l'azione obiettivata" La "obbligatorietà sorge solo quando la promessa è accettata, solo cioè dal momento in cui il destinatario di essa comincia a fare affidamento sul verificarsi del com- 
Di conseguenza bisogna che dal paradigma generale rella normatività necessariamente implicato nel discorso di chi affermi la normatività del negozio, sia possibile escludere il concetto di comando, e quindi di imperatività.

Giustamente, ad esempio, la esclusione della imperatività dal novero dei caratteri essenziali del paradigma normativo, è nel pensiero di Santi Romano ${ }^{12}$ condizione imprescindibile per l'attribuzione ai negozi di un carattere precettistico che serva, fra l'altro, a differenziarli dagli atti non negoziali ${ }^{13}$. Anche Kelsen per potere sostenere la concezione precettistica del negozio, deve opporsi alla concezione imperativistica della norma giuridica così come, ad esempio, si presenta nella formulazione di Austin ${ }^{14}$.

portamento promesso. Lo stato d'obbligo in altre parole è una creazione dell'obbligante e non dell'obbligato" (ib., p. 225).

Senonché in questo modo, a mio avviso, non si spiega come sia. possibile l'obiettivazione della volontà, come sia possibile che la volontà del promittente, pur restando volontà (sia pure volontà voluta) si proietti al di fuori della sfera volitiva del promittente per entrarenella sfera volitiva del promissario; si resta alla fine sul piano di una parificazione di promessa ed atto di alienazione con tutte le difficoltà che tale parificazione comporta. E, per ciò che concerne l'accettazione, ripugna veramente alle regole della logica giuridica il pensiero. che la promessa non accettata possa in date circostanze costituire un motivo obbligatorio? Questo pensiero è stato in realtà riconosciuto ed applicato nel diritto moderno? (Tralasciando di riferirmi a tutta una vasta letteratura sull'argomento, rimando ad una vecchia ma sempre vivida nota dell'UNGER: La promessa quale motivo obbligatorio nel diritto moderno, trad. F. ForLANI, Trieste, 1874, pp. 7-23. Vedi, anche per $i$ riferimenti alla letteratura, C. A. Funaioli. Promesse unilaterali, Siena, 1943.

12. SANTt Romano, Frammenti di un dizionario giuridico, Milano, 1947, pp. 3-30, 41-43, 64-74.

13. Intorno al problema della distinzione di atti e negozi - indipendentemente dalla soluzione da me prospettata che più non considero valida -, per la critica ad alcune dottrine più o meno legatealla concezione volontaristica del negozio, rimando al mio: Diritto $e$ scienza giuridica nella critica del concreto, Milano, 1942, pp. 153.

14. H. Kelsen, General Theory of Law and State, 3 CambridgeMass., 1949, p. 30 e ss.; trad. S. Cotta e G. Treves (Torino, 1952), p. 30 e ss. 
Per potere parlare di norma negoziale Kelsen deve depsicologizzare il paradigma normativo, eliminando da esso il carattere di comando in senso psicologico, cioè nel senso per cui l'attribuzione del carattere di imperatività alla norma giuridica in generale sia connessa alla nozione della norma giuridica come volontà.

Dal punto di vista kelseniano per depsicologizzare il paradigma normativo, bisogna dunque potere mettere fra parentesi la volontà, eliminando dalla rappresentazione del paradigma normativo l'idea di un rapporto fra la presunta volontà normativa ed $\mathrm{i}$ suoi destinatari. Sotto questo aspetto il vecchio problema dei destinatari diventa uno pseudoproblema. Il paradigma normativo si risolve formalmente ed essenzialmente nel concetto di una relazione fra un comportamento presupposto ed una conseguenza. "Se qualcuno ruba" - presupposto -, "sarà punito" - conseguenza - La rappresentazione della sanzione, come espressione di una minaccia, diventa un termine obbligato della relazione normativa. Il punto di vista tradizionale è invertito. Il "non si deve rubare", ad esempio, non può esprimere il carattere essenziale del paradigma normativo. In una descrizione esatta, formale, razionale scientifica del paradigma normativo, il "non si deve rubare" è una proposizione inessenziale e superflua rispetto al "se qualcuno ruba sarà punito" 15

4. Prescindiamo pure da tutte le difficoltà che la soluzione formale - e l'inversione kelseniana - può fare sorgere e che in essa possano rivelarsi da vari punti di vista. Limitiamoci alle sue ripercussioni per ciò che concerne la possibilità di pensare coerentemente alla essenza normativa del negozio.

Vero è che la soluzione formale elimina dal paradigma normativo quel carattere di imperatività che per le ragioni

15. KELSEN, op. cit., pp. 60-61; traduz. cit., p. 61 . 
accennate non si addice alla norma giuridica negoziale. Tuttavia anche la esclusiva riduzione del paradigma normativo nei termini di una relazione di comportamento e sanzione non va d'accordo con l'essenza normativa del negozio propugnata dal Kelsen. Le difficoltà che la soluzione formale fa sorgere sembrano proprio sotto quest'ultimo aspetto il contraccolpo teorico di quelle che vuole evitare.

Ammettendo che il paradigma normativo sia realmente riducibile nei termini di una relazione fra comportamento e sanzione, bisogna, come logica conseguenza, ammettere che solo l'organo cui spetta di ordinare la sanzione al verificarsi delle condizioni previste, sia l'effettivo destinatario dell'elemento direttivo necessariamente espresso dalle norme giuridiche ${ }^{16}$. Ma proprio in questa conseguenza, che è pur logica rispetto al suo presupposto, consiste evidentemente la ragione principale per cui non è più possibile parlare coerentemente di una norma giuridica negoziale e quindi di una norma giuridica posta in essere, ad esempio, attraverso una promessa. Infatti, pagando ad A il canone d'affitto pattuito, $B$ realizza il significato teorico e pratico della sua promessa. Appare assurdo dire che il significato pratico di una promessa si realizzi mediante l'applicazione di una sanzione conseguente al suo inadempimento, come invece si dovrebbe dire attribuendo coerentemente alla norma giuridica negoziale il paradigma normativo kelseniano.

Come può essere dunque pensato il modello normativo perchè si possa coerentemente pensare ad una norma giuridica negoziale, battendo una terza via fra le posizioni veramente significative che sono rappresentate dalla dottrina dell'autolimitazione e dal formalismo kelseniano? Per rispondere a questa domanda credo sia necessario rifarsi ad

16. KELSEN, op. cit., pp. 137-138; traduz. cit., p. 140. Vedi anche di KELSEN, La Théorie juridique de la convention in Archives de philosophie du droit et de sociologie juridique”, 1940, pp. 33-76. 
una analisi del linguaggio preccettivo che tenga calcolo di alcuni contributi recenti ${ }^{17}$.

Come regola della condotta e come precetto, una norma giuridica è una valutazione dei comportamenti. Il che non significa ancora nulla se non ci si mette d'accordo sulla differenza che passa fra proposizioni indicative (ad esempio: "l'acqua bolle a 100 gradi") e proposizioni valutative (ad esempio: "non si deve rubare").

Carattere peculiare di una proposizione valutativa è quello di esprimere una tendenza ad influenzare i comportamenti, è quindi, possiamo dire, il suo carattere direttivo. A sua volta però tale carattere direttivo non è riducibile nella forma di una relazione di causa e di effetto ${ }^{18}$.

La norma per cui $\mathrm{B}$ deve pagare ad $\mathrm{A}$, pur esprimendo una influenza sul comportamento di $\mathrm{B}$, non è correttamente pensabile come causa del comportamento di B. Al limite

17. Mi riferisco specialmente agli studi di R. M. HARE: Freedom of the Will in "Aristotelian Society", Supplementary Vol. XXV, 1951. pp. 201-216; The Language of Morals, Oxford, 1952 e ad altri autori da me citati in Responsabilità $e$ analisi del linguaggio (Riv. trim. dir. proc. civ., 1958, pp. 592-597).

18. Anche secondo R. M. HARE (The Language of Morals, cit., p. 12 e ss.), la funzione delle valutazioni pratiche di influenzare i comportamenti è concepita come determinazione causale da R. CARNAP (Philosophy and Logical Syntax, Londra, 1935, p. 24), da A. J. AYER (Language, Truth and Logic 2 Londra, 1948, p. 108), ed è particolarmente elaborata da CH. L. STEvENSON in Ethics and Language, 5 New Haven, 1950 (I ediz. 1945), e prima in Ethical Judgments and Avoidability (Mind, 1938, p. 45 e ss.) e in Persuasive Definitions (ib., p. 334 e ss.).

Sulle difficoltà suscitate dalla posizione dei ridetti autori, anche in riferimento alla questione che qui interessa, vedi $i$ miei precedenti studi: Aspetti della critica dei valori etico-giuridici nel pensiero contemporaneo in Riv. int. fil. dir., 1950, pp. 235-267 e La interpretazione del non verificabile in Il Saggiatore, 1954, pp. 69-88.

Per un contrasto ed una incoerenza interna, che si manifesta, a mio avviso, nel discorso di Stevenson, fra la sua analisi della nozione di libertà e la sua analisi delle regole della condotta, rimando alla mia relazione: Determinismo e imputabilità in Atti del II Congresso nazionale italiano di filosofia del diritto, Milano, 1956, pp. 44-47. 
dell'idea di causa, portata sul piano di considerazione dei rapporti fra le azioni umane, c'è forse proprio l'idea di comando, di costrizione, e quindi di una volontà (la volontà di chi ha voluto la norma) che si impone alla volontà dei destinatari della norma per determinarla necessariamente. Una pura determinazione causale, e quindi una influenza sui comportamenti pensata rigorosamente come determinazione costrittiva, riducibile appunto nei termini di una relazione di causa e di effetto, verrebbe ad escludere l'assenso da parte dei destinatari. Un assenso è infatti propriamente pensabile soltanto in quei casi in cui sia possibile un dissenso. La ragione dell'esistenza di norme giuridiche è legata anche alla concreta possibilità della loro violazione.

E' vero che in concreto un comportamento può essere conforme ad una regola della condotta senza che l'agente ne abbia coscienza, senza quindi che al comportamento regolare corrisponda un vero e proprio assenso da parte dell'agente. Ma ciò non toglie che scopo precipuo della formulazione di una regola sia quello di determinare la possibilità di un assenso e quindi di un consapevole comportamento ad essa conforme. Il fatto che una regola possa essere ignorata non esclude che la positiva formulazione di una regola abbia lo scopo di essere presente alla consapevolezza ed alla scelta dei destinatari.

L'assenso nei confronti dell'influenza sui comportamenti espressa da una regola della condotta, e quindi anche da una regola giuridica, è un assenso che possiamo chiamare pratico. L'assenso pratico consiste nel fatto per cui il destinatario sia disposto, nelle circostanze previste dalla regola, ad agire secondo la regola. Questo essere disposto non è un essere costretto perchè si pone come risposta ad una, sia pure implicita, domanda: Cosa devo fare? Agire secondo la direttiva espressa dalla regola, o contro la regola affrontandone le conseguenze? Il che non significa che chi assentisce praticamente debba per ciò stesso riconoscere la bontà o la giustizia di una regola, cioè la corrispondenza di essa ai suoi propri ideali di bene e di giustizia. 
A differenza dell'assenso pratico, l'assenso teoretico consiste semplicemente nel credere che una proposizione sia vera. Una proposizione è 1) indicativa, o 2) valutativa rispettivamente a seconda che, considerata nel contesto in cui si trovi espressa, 1) implichi assenso teoretico, oppure 2) implichi assenso pratico.

Una proposizione che sia concepita e posta come causa della volontà altrui indipendentemente dalla implicita postulazione di un assenso pratico, non è norma e quindi non è neanche valutazione pratica, è soltanto un puro voluto, una semplice affermazione di volontà, o una volontà che sì impone ad un'altra, o un puro imperativo. Il fatto che nel modello normativo si riveli l'implicita postulazione di un assenso pratico da parte del destinatario, è ciò che ci permette di rappresentarci e di determinare la struttura di una norma come irriducibile in termini di volontà e di comando ${ }^{19}$.

19. Dopo avere scritto la presente relazione ho potuto leggere il recentissimo saggio di E. REDENTI: Variazioni sul tema del verbo comandare in Riv. Trim. dir. proc. civ., 1959, pp. 777-794 che è, fra l'altro, un documento molto significativo di analisi del linguaggio giuridico. Ho avuto il piacere di trovare in esso un valido sostegno al punto di vista qui da me seguito.

"Le leggi in ultima analisi, dice il Redenti, sono fatte áagli uomini per gli uomini e non pex mero arbitrio, ma ispirandosi ad esigenze comunemente sentite nella vita sociale, a convinzioni, aspirazioni 0 tendenze diffuse nella massa. Ora è nella umana natura che quando si voglia ottenere per esempio che non si uccida, non si rubi, ecc., si cominci col rivolgersi prima di tutto alla "generalità di coloro che dovrebbero astenersene. Questo che non può non essere nel pensiero degli organi legislativi, dà necessariamente la sua impronta anche ai loro dettati. Perciò quando si rilevi che questi non possono effettualmente comandare ai cittadini, non si fa e non si può fare questione di indirizzo o di destinazione, ma si constata soltanto la impotenza della legge ad imporsi per via di comando in modo diretto ed immediato. Perciò io direi che i cittadini possono essere destinatari, ma non comandati o "comandabili" direttamente dalla legge. Il che non toglie che l'indirizzarsi programmaticamente a loro possa tuttavia avere una efficacia". 
La postulazione dell'assenso è in sostanza la postulazione di un elemento funzionale: precisamente nel senso che la funzione di una norma, a differenza della funzione di un puro comando, non è pensabile senza che si possa pensare a tale elemento. Ciò non toglie che possa esserci assenso da parte del destinatario anche nei confronti di un puro comando. La rilevazione del fatto per cui l'assenso effettualmente ci sia o non ci sia, è una cosa diversa dal problema concernente la necessità di postulare l'assenso come condizione della pensabilità del significato normativo di una proposizione ${ }^{20}$.

6. In conclusione, contro la prospettiva formale e l'inversione kelseniana, io credo che l'elemento caratteris-

Questi rilievi del Redenti sono preceduti da tutta una penetrante analisi degli elementi costitutivi del "comando" (nell'ordine giuridico) riducibili schematicamente, secondo la terminologia stessa usata dall'autore, alla "dualità", "programmazione", "intimazione", "subordinazione" (ib., pp. 776-785).

20. Uno dei più noti tentativi di spiegare il fondamento della obbligatorietà della promessa psicologicamente, sulla base del principio della "simpatia riflessa", è quello offerto da TH. LIPPS (Die ethischen Grunfragen,2 Amburgo e Lipsia, 1905, pp. 152-161; 167-170).

La promessa, secondo Lipps, suscita una aspettativa nel promissario il quale riceve dalle parole del promittente la volontà che il promittente gli manifesta. Il promittente, a sua volta rivive, per così dire, in se stesso l'aspettativa del promissario e con ciò si costituisce nel promittente la coscienza della obbligatorietà della sua promessa. C'è quindi un rapporto riflesso di simpatia per cui la volontà del promittente si riflette nella "coscienza" del promissario e ritorna obiettivata al promittente costituendo in lui la coscienza dell'obbligo (op. cit., p. 167).

Lipps in sostanza parte da una concezione puramente volontaristica della promessa e cerca di spiegare proprio ciò che, dal punto di vista volontaristico, è, secondo me, impossibile spiegare e cioè la presunta obiettivazione della volontà del promittente. Basta proprio che io sappia che un altro ha avuto conoscenza della mia volontà perchè il contenuto della mia volontà acquisti nei miei confronti il significato obiettivo di un obbligo?

Reinach osserva giustamente (op. cit., p. 74-75) che, secondo il punto di vista di Lipps, la promessa fatta a Tizio di agire nell'interesse 
tico di una proposizione normativa - in quanto proposizione valutativa, considerata nel contesto di cui fa parte e nelle sue implicazioni d'uso - consista nella espressione di una tendenza ad influenzare e dirigere i comportamenti; ma, contro la prospettiva volontaristica ed imperativistica, escludo che la influenza direttiva della norma sui comportamenti possa essere identificata nei termini di una relazione di causa e di effetto, in quanto che l'assenso pratico $\grave{e}$, secondo me, il postulato funzionale di tale influenza ${ }^{21}$.

di Caio dovrebbe fare sorgere un diritto di Tizio nei confronti del promittente e non di Caio. Il che sarebbe anche in contrasto col fatto per cui Lipps è costretto a riconoscere che la pretesa dell'adempimento è più forte in chi ha l'interesse. Inoltre, contro Lipps, Reinach dimostrò che spesso obblighi, pretese e diritti si costituiscono indipendentemente da ogni situazione psicologica "interna" consistente in volontà e sentimenti.

In conclusione è fallito il tentativo offerto dalla psicologia di Lipps (autore anche di un libro intitolato: Leitfaden der Psychologie, Lipsia, 1903) di spiegare il fondamento dell'obbligo attraverso il presunto riflettersi - o "simpatia riflessa" - della volontà del promittente nella coscienza del promissario e della aspettativa del promissario nella coscienza del promittente: questo presunto gioco di riflessioni simpatetiche non serve come spiegazione. Ciò non toglie tuttavia, secondo me, che una diversa nozione di partecipazione simpatetica possa servire ad esprimere la struttura del processo valutativo. Infatti, se, come io cerco di sostenere, ogni norma contiene una valutazione pratica ed ogni valutazione pratica implica una tendenza ad influenzare i comportamenti nella quale - e nel senso ridetto — è postulato l'assenso da parte dei destinatari, ne deriva che l'atto in cui si concreta una valutazione pratica deve potere essere in qualche modo concepibile come atto di partecipazione alla situazione reale od ipotetica dei destinatari. Su ciò rimando ai miei precedenti studi intorno al problema della partecipazione simpatetica intesa come "simpatia indiretta": La simpatia nella morale e nel diritto. Aspetti del pensiero di A. Smith. ristampa, Torino, 1958; Utilità e limiti della sociologia nella valutazione in termini di giustizia distributiva inAut Aut, 1954, n. 21, pp. 196-203; Giustizia distributiva e simpatia in Riv. int. fil. dir., 1954, pp. 712-725.

21. Una discussione adeguata della analisi del linguaggio precettivo compiuta da HARE non può essere fatta qui. Io accetto in par- 
Di conseguenza, ripeto, se la volontà del promittente è concepita come creatrice di una norma, non può essere per ciò stesso concepita come causa del comportamento futuro del promittente consistente nell'adempimento della promessa. La volontà dell'adempimento non è causata dalla volontà che ha posto in essere la promessa. La volontà dell'adempimento non è l'effetto della volontà passata del promittente. Si evita così l'assurdo concetto dell'autolimitazione della volontá. Fra la volontá di promettere e la volontà di adempiere c'è la norma creata dalla volontà di promettere. Ed inoltre da un lato la norma posta in essere dalla volontà di promettere e, d'altro lato, la volontà di adempiere non sono a loro volta concepibili come termini di una relazione di causa e di effetto, propio perché la volontà di adempiere la promessa è una scelta, e non semplicemente un effetto. E' una scelta fra due possibilità: adempimento o inadempimento, implicanti rispettiva-

te il punto di vista dell'autore per ciò che concerne 1" assenso" nella determinazione della differenza fra proposizioni indicative e proposizioni precettive (The Language of Morals, cit., p. 18 e ss. e Freedom of the Will, cit., p. 215). Ma, fra l'altro, non mi sembra vero ciò che egli cerca di dimostrare contro Carnap, Ayer e Stevenson negando che la funzione di un comando sia quella di determinare il destinatario ad agire nel modo espresso dal comando medesimo (The Language of $\mathrm{Mo-}$ rals. cit., p. 13). Si può proprio, senza previamente alterare il significato comune della parola "comandare" così come si presenta nei più diversi contesti, parlare, ad esempio, di un volere comandare che non implichi un volere essere obbedito? $\mathbf{E}$ corretto parlare di un volere comandare che si ponga come tendenza ad "istruire" e a "guidare" le azioni dei destinatari senza peraltro porsi come tendenza ad influenzare $i$ comportamenti dei destinatari ed a persuaderli ad agire nel senso espresso dal comando (Freedom of the Will, cit., p. 214) ? Mi sembra che nel pensiero di Hare ci sia un equivoco costituito dal fatto di assumere indebitamente le nozioni di "influenza sui comportamenti" e di "persuasione" come esclusivamente riducibili nei termini di una "determinazione causale". un equivoco che probabilmente nasce dalla tensione polemica dell'autore nei confronti del punto di vista che egli attribuisce per l'appunto, come, dicevo, a Carnap, Ayr e Stevenson. 
mente un atto di assenso o un atto di dissenso nei confronti dell'influenza sui comportamenti espressa dalla norma negoziale.

Come si è visto, secondo l'inversione operata dal Kelsen rispetto al punto di vista tradizionale, le formulazioni del tipo "non si deve rubare" sarebbero superflue ed inessenziali, in una descrittiva scientifica del paradigma normativo, rispetto alle formulazioni del tipo "se qualcuno ruba sarà punito". Perciò la norma per cui un debitore deve pagare, non sarebbe diretta al debitore, ma sareōbe dî̃retta, in maniera condizionata dall'inadempimento del debitore, all'organo che deve applicare la sanzione. Ma così per l'appunto si perde, nel senso che già si è visto, il significato direttivo della norma come valutazione pratica e, a mio avviso, non si può coerentemente parlare di norma giuridica negoziale ${ }^{22}$.

Perchè sia possibile parlare coerentemente di norma negoziale bisogna invece potere dire - come credo si possa dire se è accettabile il punto di vista qui proposto - che la norma è diretta al debitore e che la sua influenza direttrice è condizionata dalla credenza che la sanzione sarà applicata in caso di inadempimento.

La sanzione è sì evidentemente il mezzo che la regola giuridica presuppone, a differenza di altre regole pratiche, affinché sia esercitata la sua influenza sui comportamenti; ma perchè si possa parlare di norma giuridica negoziale l'influenza direttrice della norma non deve essere identificata con il mezzo con cui si esercita e che pur tuttavia necessariamente implica. (L'influenza direttrice di un precetto giuridico è insomma condizionata dalla credenza che la sanzione sarà applicata o anche forse dal fatto che un

22. A Ross che pure avendo una sua generale prospettiva teorica personale, sembra accettare l'inversione kelseniana (On Law and Justi$c e$, Londra, 1958, p. 52) è più coerente del Kelsen quando si astiene da una concezione normativa del negozio (ib., p. 224). 
precetto faccia parte di un sistema di precetti per la maggior parte sanzionabili) ${ }^{23}$.

La descrizione di questo rapporto di imprescindibile implicazione fra norma negoziale e sanzione presuppone poi evidentemente la descrizione delle relazioni fra norma negoziale ed altre norme dell'ordinamento giuridico positivo in cui la norma negoziale si trovi inserita.

23. Secondo H. L. A. HART (Positivism and The Separation of Law and Morals in Harvard Law Review, 1958, p. 621), "vi sono molte cose che non sono vere se vengono riferite alle norme giuridiche considerate separatamente" rispetto al sistema di norme di cui fanno parte, "ma che sono vere ed importanti se riferite in generale al sistema legale di cui fanno parte, considerato come un tutto". Ciò vale anche, secondo l'autore, per la "connessione" fra norme e sanzioni.

Fra le più recenti e vivaci discussioni in tema di sanzione vedi nella letteratura italiana F. ALLORIO, Osservazioni critiche sulla sanzione (estratto da Studi in onore di F. Messineo, v. IV), Milano, 1958, pp. 3-50 e D. BARBero, Polemiche sulla sanzione (estr. da Riv. dir. civ., 1956), Padova, pp. 3-24. 